\title{
Real-Time Driver's Biological Signal Monitoring System
}

\author{
Jin Hong Ju, Young Jun Park, Jaehee Park*, \\ Boon Giin Lee, Jaecheon Lee ${ }^{1}$ and Jea Yeol Lee ${ }^{2}$ \\ Department of Electronic Engineering, Keimyung University, \\ 1095 Dalgubeol-daero, Dalsoe-Gu, Daegu 704-701, Korea \\ 'Department of Mechanical \& Automotive Engineering, Keimyung University, \\ 1095 Dalgubeoldaero,Daegu 704-701, Korea \\ ${ }^{2}$ Hwajin, Donamgongdangil 16, Yeongcheon, Gyungbuk, Korea
}

(Received July 14, 2014; accepted December 8, 2014)

Key words: biological signals, respiration, gripping force, PPG, driver's state of vigilance

A real-time system is presented for monitoring the driver's biological signals, including respiration, the gripping force, and a photoplethysmogram (PPG). The proposed monitoring system is composed of three biological sensors, an interface circuit, a data acquisition (DAQ) board, and a personal computer (PC). The respiration and gripping force signals are obtained from pressure sensors attached to the seat belt and steering wheel, respectively. The PPG signal is acquired from a PPG sensor that consists of a light-emitting diode (LED) and a phototransistor (PT) attached to the steering wheel. The characteristics of the biological sensors used in the proposed monitoring system are comparable to those of proven sensors. A real-time monitoring program used to display and analyze the three biological signals is also developed using LabView, and used to calculate and display the peak amplitude and interval of the PPG and respiration signals, along with the driver's gripping force applied to the steering wheel. The results show that the proposed real-time driver's biological signal monitoring system can be effectively utilized for measuring a driver's state of vigilance.

\section{Introduction}

The number of car accidents due to driver inattention continues to increase, and drowsiness and fatigue have become one of the major causes of serious traffic accidents. According to the U.S. National Highway Traffic Safety Administration (NHTSA), falling asleep while driving is responsible for at least 100000 automobile crashes annually, resulting in annual averages of roughly 40000 nonfatal injuries and 1550 fatalities. ${ }^{(1)}$ The National Sleep Foundation has also reported that $60 \%$ of adult drivers have driven while feeling drowsy, and $37 \%$ have even actually fallen asleep at the wheel. ${ }^{(2)}$

${ }^{*}$ Corresponding author: e-mail: jpark@kmu.ac.kr 
For this reason, various methods have been developed for monitoring the state of vigilance of drivers to avoid accidents related to driver drowsiness. For example, some methods monitor the driver's state of vigilance on the basis of driving performance, ${ }^{(3)}$ where the vehicle lane position, microsteering movements, steering wheel angle, and steering adjustment time can be used to estimate the driver's fatigue. However, while performance-based monitoring methods have the advantages of being inexpensive and immune to problems associated with the environment and weather, such as low-light and rainy conditions, their monitoring accuracy is low as they monitor the driver's state of vigilance indirectly. Thus, monitoring methods based on eye activity using video cameras have been developed to indicate the driver's state of vigilance. However, while such video-camera methods, in particular those which monitor the percentage of eyelid closure (PERCLOS), are already widely accepted for monitoring a driver's state of vigilance, they have difficulty capturing reliable images when the environmental light is highly variable, ${ }^{(4)}$ and significant computer power is needed to process the video in real time. Thus, the most accurate monitoring methods use physiological signals, such as brain waves and heart rate, pulse rate, and respiration rate signals. ${ }^{(5)}$ Although brain waves are the best physiological signal to evaluate a driver's state of vigilance, they are very difficult to obtain during driving. The next best biological signal is an electrocardiogram (ECG) signal with heart rate (HR) and heart rate variability (HRV) information. A number of psychophysiological studies have determined that the low frequency $(0.04-0.15 \mathrm{~Hz})$ to high frequency $(0.15-0.4 \mathrm{~Hz}) \mathrm{HRV}$ component ratio decreases when a person becomes drowsy. ${ }^{(6)}$ Thus, an ECG monitoring system ${ }^{(7)}$ for monitoring driver drowsiness has already been reported, along with a driver drowsiness monitoring system utilizing three biological signals, including ECG and pulse oximetry signals. ${ }^{(8)}$ However, these monitoring systems are not acceptable in practice, as the ECG signal is strongly dependent on environmental noises, such as vibration and temperature variation. Accordingly, this paper presents a real-time driver monitoring system that uses biological signals that are less sensitive to automotive environmental noises. Such biological signals include respiration, the gripping force, and a photoplethysmogram (PPG). When compared with other monitoring systems, the proposed monitoring system can accurately monitor a driver's state of vigilance through the use of biological signals that are less sensitive to automotive environmental noises.

\section{Real-Time Driver's Biological Signal Monitoring System}

\subsection{Real-time monitoring system}

The biological signals of a driver are a good indicator of his state of vigilance. Nonetheless, only one biological signal is still not sufficient to accurately monitor a driver's state of vigilance. Therefore, the proposed real-time monitoring system utilizes three biological signals, namely, respiration, a PPG, and the gripping force on the steering wheel. These biological signals are all easily obtained, especially in an automobile.

The driver's biological signal monitoring system (Fig. 1) proposed in this paper consists of two pressure sensors to measure the respiration and gripping force, a PPG 


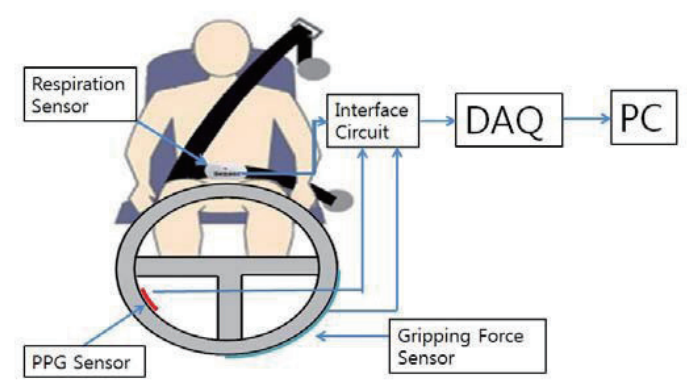

Fig. 1. (Color online) Schematic diagram of proposed real-time monitoring system.

sensor with a light-emitting diode (LED) and a phototransistor (PT), an interface circuit, a 10-bit digital acquisition (DAQ) board, and a personal computer (PC). The gripping force and PPG sensors are attached to the steering wheel. The respiration sensor is bonded to the abdomen part of the seat belt. The biological signals from the sensors are filtered and amplified by the interface circuit, converted to digital signals at the DAQ board, and then sent to the PC, where they are analyzed and displayed.

\subsection{Respiration}

The respiration signal is an important and easy-to-measure indicator, and a correlation has recently been found between respiration and drowsiness. ${ }^{(9)}$ In the proposed monitoring system, the respiration signal is obtained using a respiration pressure sensor bonded to the seat belt. As the driver inhales and exhales, this changes the belt length and applies pressure to the respiration sensor bonded to the belt. The respiration signal is then acquired from the resistance variations of the pressure sensor. An electric circuit (Fig. 2) is used to convert the resistance variations of the pressure sensor into an electrical respiration signal. This electric circuit consists of a low-pass filter with a cutoff frequency of $0.5 \mathrm{~Hz}$, a high-pass filter with a cutoff frequency of $0.2 \mathrm{~Hz}$, and an amplifier with an amplification of 15. The pressure sensor (Interlink Electronics FSR408) used in the monitoring system is a piezoelectric pressure sensor attached to a seat belt (Fig. 3). The resistance of the sensor is inversely proportional to the applied pressure. Figure 4 shows the respiration signal from the pressure sensor attached to a seat belt, while Fig. 5 shows the respiration signal from a Biopac respiration measurement device (MP150WSW). These figures show that the pressure sensor attached to a seat belt has a similar response to a Biopac respiration measurement device, confirming its effectiveness in obtaining a respiration signal.

\section{$2.3 P P G$}

A PPG is an optically obtained plethysmogram, a volumetric measurement of an organ. Generally, a PPG signal is obtained using a PPG sensor with an LED and PT. The light from the LED is shone onto the skin, and the amount of light reflected to the PT is then measured. The amount of reflected light depends on the volume change of the blood vessels induced by the pressure pulse of the cardiac cycle. ${ }^{(10)}$ The signal derived 

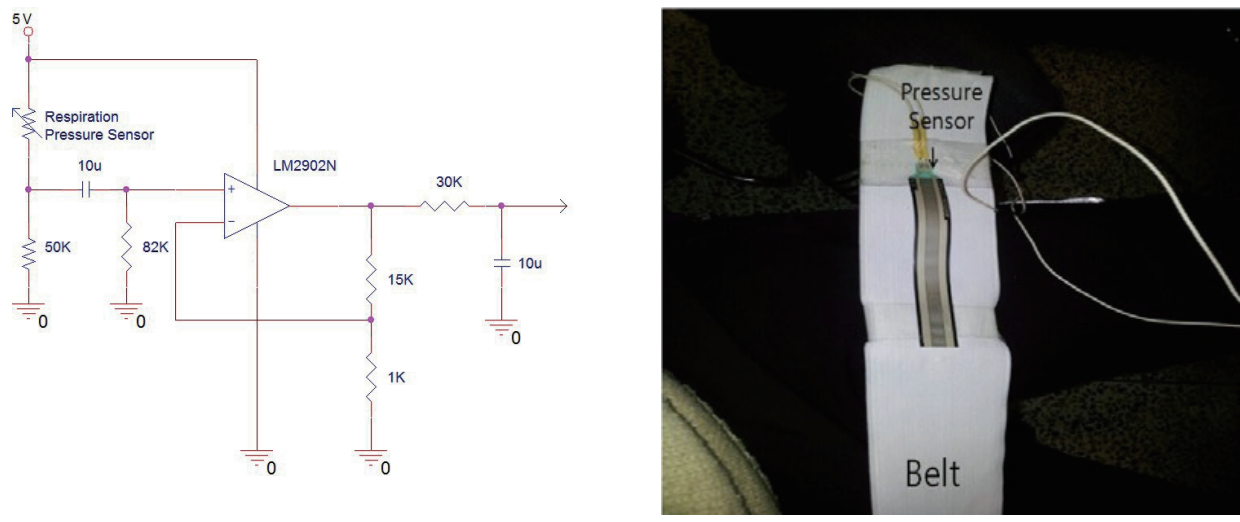

Fig. 2 (left). (Color online) Respiration sensor circuit.

Fig. 3 (right). (Color online) Respiration pressure sensor attached to seat belt.

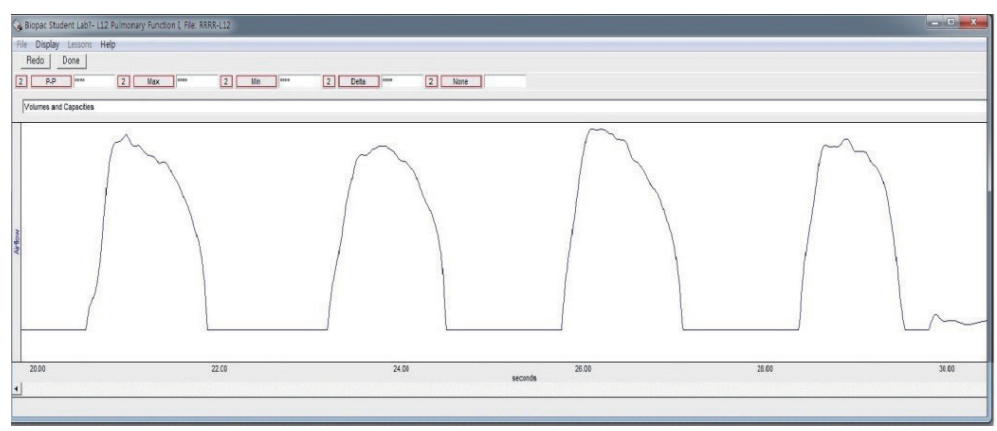

Fig. 4. (Color online) Respiration signal from pressure sensor.

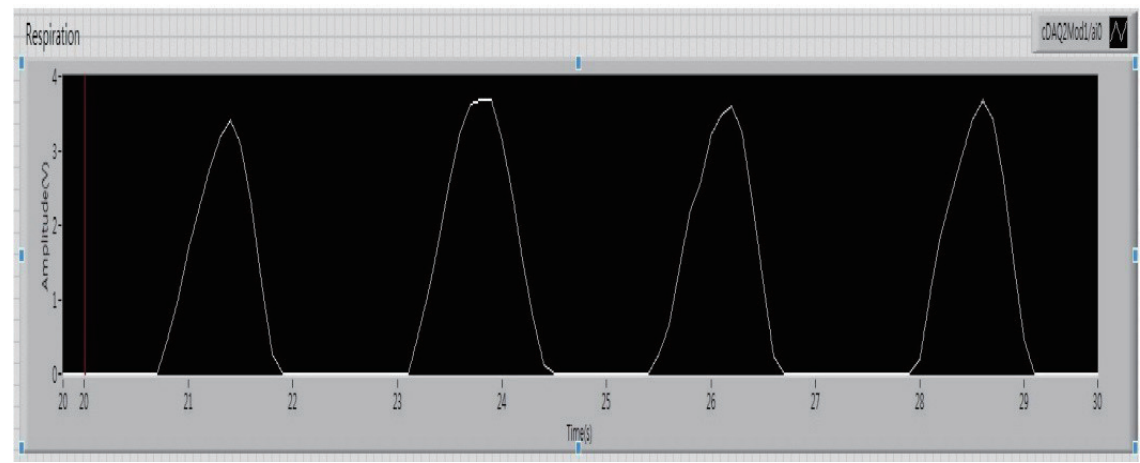

Fig. 5. Respiration signal from Bio-Pac (MP150WSW). 
from the PPG sensor is then processed to obtain information on the HR and HRV. In the proposed monitoring system, the PPG sensor is attached to the steering wheel (Fig. 6), and an electric circuit (Fig. 7) is used to acquire the PPG signal from the PPG sensor. The electric circuit is composed of an LED (Kodenshi EL-23G), PT (Kodenshi ST$23 \mathrm{G})$, a low-pass filter with a cutoff frequency of $48 \mathrm{~Hz}$, a high-pass filter with a cutoff frequency of $0.16 \mathrm{~Hz}$, and an amplifier with an amplification of 300 . This electric circuit converts the optical PPG signal into an electric PPG signal that is then sent to a PC. Figure 8 shows the PPG signal waveforms from the PPG sensor in the proposed realtime monitoring system and from a PPG test kit (EK-604D, Beijing Choice Electronics), respectively. The upper waveform is the signal waveform from the pressure sensor, while the bottom waveform is the signal waveform from the PPG test kit. These figures show that the PPG sensor attached to the steering wheel has similar characteristics to a certified PPG test kit, confirming its effectiveness for obtaining a driver's PPG signal.
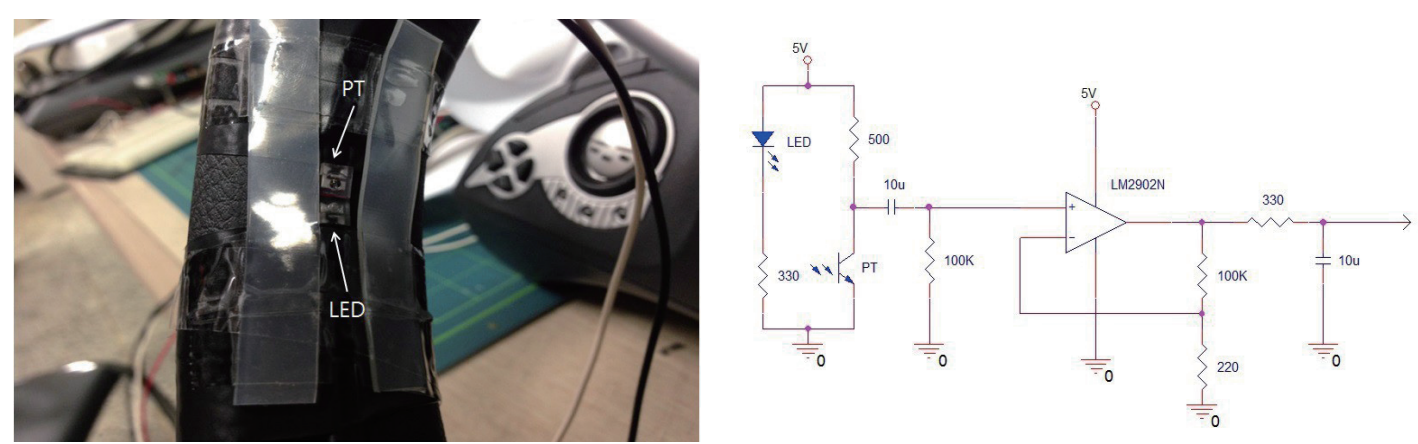

Fig. 6 (left). (Color online) PPG sensor attached to steering wheel.

Fig. 7 (right). (Color online) PPG sensor circuit.

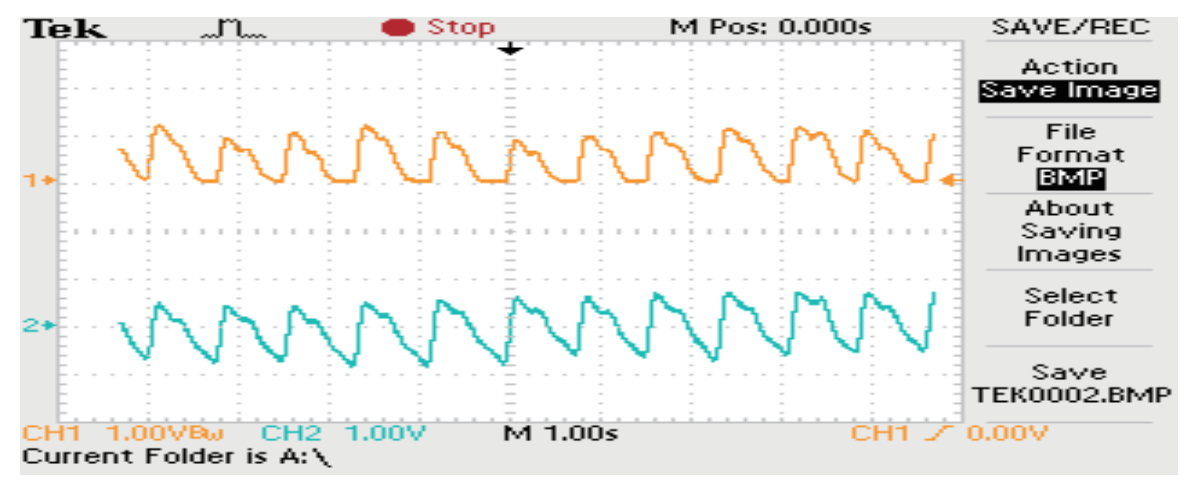

Fig. 8. (Color online) PPG signals (upper waveform: PPG sensor attached to steering wheel; bottom waveform: EK-604D). 


\subsection{Gripping force}

The proposed method of monitoring the driver's gripping force on the steering wheel is an economical method. The gripping force on the steering wheel generally decreases when a driver becomes drowsy, as the driver's muscles become increasingly relaxed. ${ }^{(11,12)}$ The gripping force signal is derived from a pressure sensor attached to the steering wheel, as shown in Fig. 9. The pressure sensor used to monitor the gripping force is a piezoelectric pressure sensor (Interlink Electronics FSR408). The resistance of the pressure sensor decreases as the gripping force on the steering wheel increases. The resistance changes are then converted into an electric signal using a voltage follower circuit (Fig. 10). Finally, the gripping force on the wheel is calculated using eq. (1) derived from the characteristic graph of the pressure sensor. ${ }^{(13)}$

$$
G(V)=\beta\left(\frac{910 \times 5-910 V}{V}\right)^{\frac{1}{\alpha}}
$$

Here, $G(V)$ is the gripping force on the wheel, $\alpha$ is approximately $0.7379, \beta$ is approximately $1.6 \times 10^{7}$, and $V$ is the voltage across the pressure sensor. The measurement range of the gripping force is from $20 \mathrm{~g}$ to $10 \mathrm{~kg}$. The measurement sensitivity was calculated to be approximately $13 \mathrm{~g}$. Figure 11 shows the waveform of the gripping force on the steering wheel. The results demonstrate that attaching the pressure sensor to the steering wheel is effective in measuring the driver's gripping force on the steering wheel.

\subsection{Monitoring software}

The biological signals obtained from the three sensors are transmitted to the personal computer through the interface circuit and DAQ board. These signals are then analyzed and displayed on the PC monitor. The driver's biological signal monitoring software is developed using LabView. Figure 12 shows the monitoring panel that displays the
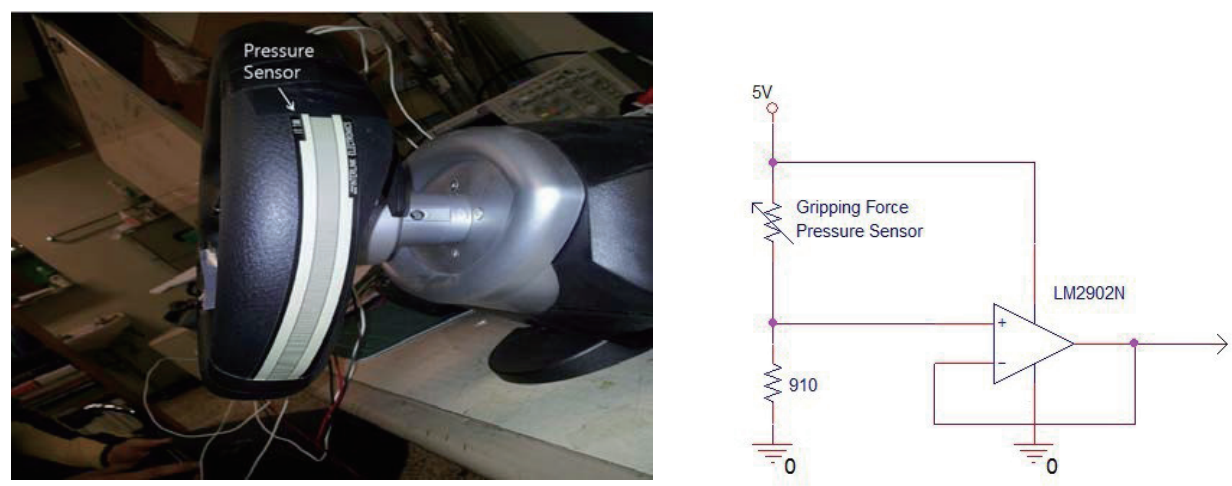

Fig. 9 (left). (Color online) Gripping force pressure sensor attached to steering wheel. Fig. 10 (right). (Color online) Gripping force sensor circuit. 


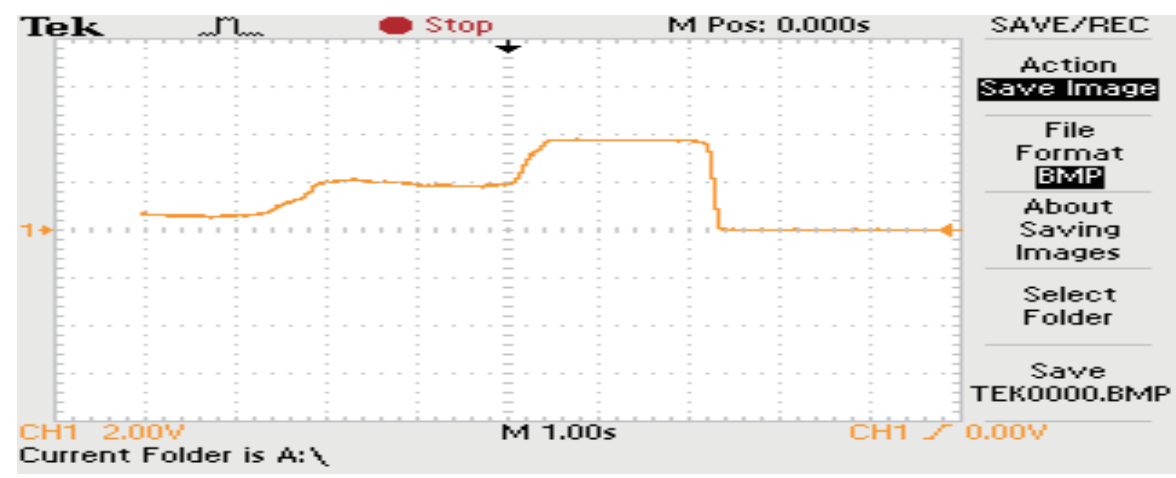

Fig. 11. (Color online) Grapping force signal.

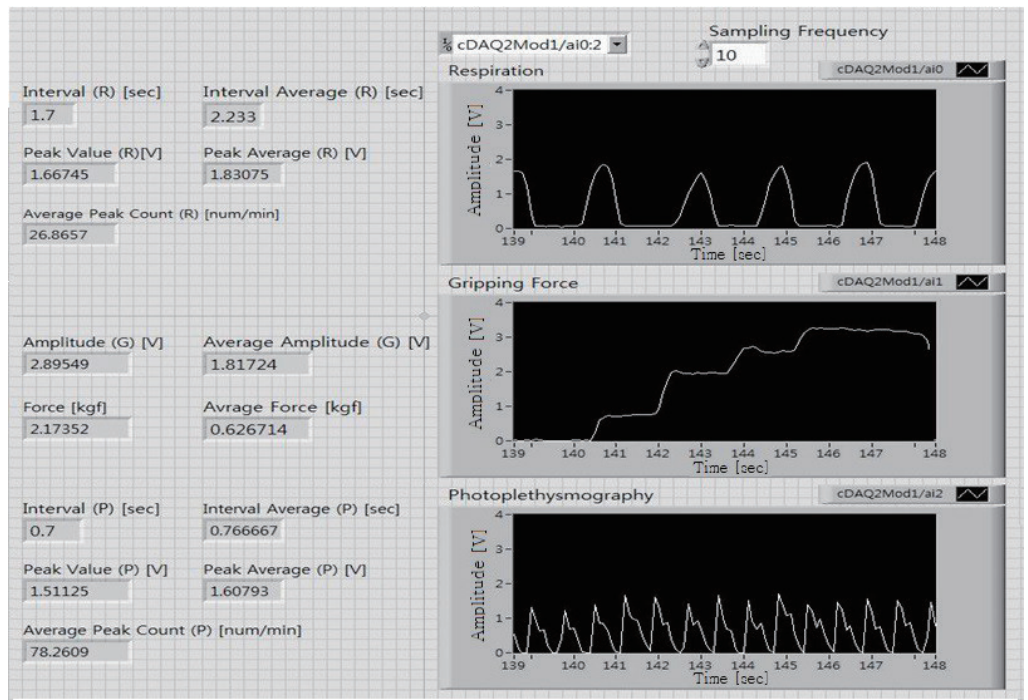

Fig. 12. Monitoring panel.

biological signals and all the information related to the three biological signals. The respiration signal (right) and information (left) about the respiration signal are displayed at the top of the monitoring panel, and include the instantaneous peak interval, average peak interval, instantaneous peak respiration signal amplitude, average peak respiration signal amplitude, and number of peaks per minute. All this information is obtained and calculated from the respiration signal. On the other hand, the gripping force signal (right) and all the information (left) on the driver's gripping force applied to the steering wheel are displayed at the center of the monitoring panel, and include the instantaneous force signal amplitude, average force signal amplitude, instantaneous gripping force, and average gripping force. This information is mostly obtained from the gripping force 
signal, while the instantaneous and average gripping forces are calculated using eq. (1). Finally, the PPG signal (right) and all the information (left) on the driver's PPG signal are shown at the bottom of the monitoring panel, and include the instantaneous peak interval, average peak interval, instantaneous peak PPG signal amplitude, average peak PPG signal amplitude, and number of peaks per minute. All this information is obtained from the PPG signals. The peak positions of the respiration and PPG signals are calculated using a noise threshold digital peak detection algorithm. ${ }^{(14)}$ The averaging time is $1 \mathrm{~min}$ and the sampling frequency is $10 \mathrm{~Hz}$. Thus, it is anticipated that the proposed real-time driver's biological signal monitoring system using respiration, the gripping force, and PPG information can be effectively utilized in a driver drowsiness detection system.

\section{Discussion and Conclusions}

In this study, a new real-time system for accurately monitoring a driver's state of vigilance despite the harsh monitoring conditions of an automobile is proposed. The driver's biological signals used for the real-time monitoring are biological signals that are less sensitive to automotive environmental noises, and include respiration, the gripping force, and a PPG. The real-time monitoring system is composed of three biological sensors, an interface circuit, a DAQ board, and a PC. The sensors used to obtain the respiration signal and gripping force applied to the wheel are piezoelectric pressure sensors attached to the seat belt and steering wheel, respectively. Experimental results show that the respiration pressure sensor has a similar response to a certified Biopac respiration measurement device, confirming its effectiveness in measuring the driver's respiration signal. Moreover, the gripping force measurement sensor is shown to be effective for measuring the driver's gripping force applied to the steering wheel. The PPG sensor includes an LED and a PT attached to the steering wheel. Experimental results also show that the PPG sensor has comparable characteristics to a certified PPG test kit, confirming its effectiveness in monitoring the driver's PPG signal. The monitoring program used to display and analyze the driver's biological signals is developed using LabView. The driver's biological signals, including the respiration, gripping force, and PPG, along with other information (e.g., peak interval, peak amplitude, and gripping force) on the driver's biological signals, are displayed on the monitoring panel. The results show that the proposed system can be utilized to monitor a driver's state of vigilance. A degradation in the performance of the proposed monitoring system occurred when changing the installation locations of the sensors. Thus, the installation methods and locations of these sensors still need to be studied. In addition, the relationship between the driver's state of vigilance and the three biological signals also needs to be investigated further. A digital processing algorithm can then be developed to allow the proposed real-time driver's biological signal monitoring system to be effectively utilized in a driver drowsiness detection system. 


\section{Acknowledgements}

This work was supported by the Ministry of Knowledge Economy (MKE) and Korea Institute for Advancement of Technology (KIAT) through the Center for Automotive Mechatronics Parts (CAMP) at Keimyung University.

\section{References}

1 L. Bergasa, J. Nuevo, A. Sotelo, R. Barea and M. Lopez: IEEE Int. Transp. Syst. 7 (2006) 63.

2 M. Rosekind: Sleep Med. 6 (2005) S21.

3 A. Eskandariam and A. Mortazavi: Proc. Intelligent Vehicles Symp., L. Guvenc (IEEE, Istanbul, 2007) p. 553.

4 J. McCall and M. Trivedi: IEEE Int. Transp. Syst. 7 (2006) 20.

5 Q. Wang, J. Yang, M. Ren and Y. Zheng: Proc. 6th World Conf. Intelligent Control and Automation, J. Song, Y. Ho and T. Tran (IEEE, Dalian, 2006) p. 8587.

6 S. Hu, R. Bowlds, Y. Gu and X. Yu: Proc. 31st Annual International IEEE EMBS Conf., B. He (IEEE, Minnesota, 2009) p. 2312.

7 T. Matsuda and M. Makikawa: Proc. 30th Annual International IEEE EMBS Conf., B. He (IEEE, Vancouver, 2008) p. 1315.

8 H. Rosario, J. Solaz, N. Rodriguez and L. Bergasa: IET Intel. Transport Syst. 4 (2010) 280.

9 J. Park, J. Kim and J. Lee: J. Sen. Sci. Tech. 23 (2014) 142.

10 K. Shelley, D. Jablonka, A. Awad, R. Stout, H. Rezkanna and D. Silverman: Anesth. Analg. 103 (2006) 372.

11 Y. Lin, H. Leng, G. Yang and H. Cai: IEEE Sensors J. 7 (2007) 790.

12 J. Park: Opt. Eng. 50 (2011) 020501.

13 Interlink Electronics: Homepage of Interlink Electronics, http://www.interlinkelectronics. com/FSR408.php (accessed in July 2014).

14 V. Jordanov, D. Hall and M. Kastner: Proc. IEEE Unclear Sci. Symp., S. Metzler (IEEE, Norfolk, 2002) p. 140. 